Running head: Personality and delay discounting in fMRI

\title{
Intelligence and Extraversion in the neural evaluation of delayed rewards.
}

\author{
Claudia Civai $^{\mathrm{a}^{*} \text {, }}$, Daniel R. Hawes ${ }^{\mathrm{b} 2}$, Colin G. DeYoung ${ }^{\mathrm{c}}$, Aldo Rustichini ${ }^{\mathrm{a}}$ \\ ${ }^{a}$ University of Minnesota, Department of Economics \\ 4-101 Hanson Hall, \\ $19254^{\text {th }}$ St South \\ Minneapolis, MN, 55455, U.S.A. \\ ${ }^{\mathrm{b}}$ University of Minnesota, Department of Applied Economics \\ 1994 Buford Ave, \\ Falcon Heights, MN, 55108, U.S.A. \\ ${ }^{c}$ University of Minnesota, Department of Psychology \\ 75 E River Rd, \\ Minneapolis, MN, 55455, U.S.A.
}

*Corresponding author:

Claudia Civai

Radboud University, Donders Institute for Brain, Cognition and Behaviour

Centre for Cognitive Neuroimaging

Kapittelweg 29,

6525 EN Nijmegen,

The Netherlands

E-mail: c.civai@donders.ru.nl

Phone: +31618843195

\footnotetext{
${ }^{1}$ Present address: Radboud University, Donders Institute for Brain, Cognition and Behaviour, Centre for Cognitive Neuroimaging, Kapittelweg 29, 6525 EN, Nijmegen, The Netherlands.

${ }^{2}$ Present address: Universität Hamburg, Fakultät für Wirtschafts- und Sozialwissenschaften, Von-Melle Park 5 (Raum 3076), 20146 Hamburg, Germany.
} 


\begin{abstract}
Temporal discounting (TD), the preference for earlier, smaller rewards over delayed, larger rewards, is a pervasive phenomenon that covaries with Big Five personality traits and Intelligence (IQ). This study provides novel insight by identifying correlates for IQ and Extraversion in the neural representation of TD preferences. An intertemporal choice task was employed, where offers were sequentially presented, distinguishing between one evaluation phase (first offer is presented) and one comparison phase (second offer is presented and values are compared). IQ correlated with responses of caudate nucleus to the subjective values of the offers, suggesting a role of cognitive abilities in modulating reward responses. Extraversion correlated with the strength of functional connectivity of a reward evaluation network centered on ventromedial prefrontal cortex.
\end{abstract}

Keywords: Intelligence; Extraversion; temporal discounting; decision preference; functional connectivity; choice; evaluation network; caudate nucleus; VMPFC. 


\section{Introduction}

The consequences of our decisions are not always immediate. Many decisions specifically require us to evaluate current options for their future outcomes. When future outcomes are positive - that is, rewards - human and non-human animals reveal a preference, all else being equal, for earlier delivery (see Frederick et al., 2002 for a review). Later rewards are thus discounted relative to sooner rewards. Relatively stable individual differences in this temporal discounting (TD) tendency are linked to a number of life outcomes. Preference for larger delayed rewards over smaller immediate rewards (i.e., reduced TD) has been shown to predict both income and academic performance (Duckworth \& Seligman, 2005; Duckworth, Tsukayama, \& Kirby, 2013; Evans \& Rosenbaum, 2008)). TD also serves as a risk factor for externalizing problems, including substance abuse disorders, aggression, and delinquency (Krueger, Caspi, Moffitt, White, \& Stouthamer-Loeber, 1996; Verdejo-García, Lawrence, \& Clark, 2008). A better understanding of the underlying factors contributing to stable individual differences in TD can contribute critically to understanding human decision-making, as well as potentially lead to improvements in both clinical treatment and social intervention.

A promising pathway toward understanding the source of individual variations in TD is to link it simultaneously to well-studied, broad dimensions of psychological variation and at the same time to associated underlying neural substrates. Variations in TD, as modeled by decision theory, can be interpreted and understood within the broader frame of personality theory, and the addition of the neural underpinnings allows a deeper understanding of the decision-making mechanisms. This extension aids us in attributing the sources of the individual differences in choice to deeper individual differences in information processing and control functions (DeYoung, 2015), clarifying for instance the roles of Extraversion and intelligence. Toward this 
end, we carried out a study of the neural basis of TD in relation to the Big Five personality traits and intelligence. TD is robustly associated with intelligence (IQ); meta-analysis has estimated the correlation at $r=-.23$ (Shamosh \& Gray, 2008). TD has also been linked to Big Five traits (most often Extraversion), but less strongly (Hirsh, Guindon, Morisano, \& Peterson, 2010; Hirsh, Morisano, \& Peterson, 2008; Ostaszewski, 1996). One very large study $(N=5,888)$ found that TD was associated positively with Extraversion and Neuroticism and negatively with Conscientiousness and Openness/Intellect (and unrelated to Agreeableness), but the strongest effect (for Extraversion) was only equivalent to a correlation of .10 (Mahalingam, Stillwell, Kosinski, Rust, \& Kogan, 2014). Nonetheless, theories of the psychological functions underlying the Big Five render these associations intelligible (e.g., DeYoung, 2015). Extraversion appears to reflect sensitivity to reward (Depue \& Collins, 1999), which may increase the desirability of sooner rewards relative to later rewards, whereas Neuroticism appears to reflect sensitivity to threat (Gray \& McNaughton, 2000), which may increase the aversiveness of waiting for a delayed reward (in part due to the threat posed by uncertainty; Hirsh \& Inzlicht, 2008). Conscientiousness reflects self-discipline and the tendency to resist distraction and act deliberatively, which are clearly conceptually opposed to TD. Openness/Intellect is the Big Five trait most strongly related to intelligence, which probably explains its association with TD (which was the weakest of the four effects found by Mahalingam et al., 2014).

Research on the neurobiology of TD provides additional guidance for developing hypotheses regarding TD's link to the Big Five and intelligence. In-depth research has been carried out on the neural processes that subserve intertemporal decision-making (see Peters \& Buchel, 2011, for a review). Most of this research has been organized around the differentiation between two systems thought to be central to value-based decisions. The first is a core evaluation 
network, including ventral striatum and ventromedial prefrontal cortex (VMPFC), which represents neural subjective value signals (McClure et al., 2004; Kable \& Glimcher, 2007; for a review on VMPFC and the representation of unified subjective value see Rangel \& Clithero, 2013). The second is a modulating control network, including dorsal and ventral lateral prefrontal areas and the dorsal anterior cingulate (ACC), which engages in maintaining information in working memory and inhibiting prepotent responses (Kable \& Glimcher, 2009; Hare, Camerer, \& Rangel 2009; Figner et al., 2010). Recent research showed that functional connectivity between VMPFC and dorsolateral prefrontal cortex (DLPFC) plays an important role in determining intertemporal choice. A study by Hare, Hakimi, \& Rangel (2014) found that the DLPFC was more strongly connected to the VMPFC at the moment of choice and, in particular, during selection of later rewards, suggesting that DLPFC may contribute to revealed preferences by modulating VMPFC value signals during decision-making. This interpretation is supported by brain stimulation data (Figner et al., 2010), which demonstrates that delivering TMS over the DLPFC and disrupting its activation decreases the choice of delayed rewards without changing participants' ratings of how much they like those same rewards.

These two brain systems involved in TD clearly implicate the known neural substrates of Extraversion and Intelligence, precisely the two basic traits that appear to be most strongly linked to TD behaviorally. Many studies have now shown that Extraversion is related to the sensitivity of the dopaminergic reward system that is the core of the evaluation network (Depue \& Fu, 2013; DeYoung, 2013; Wacker \& Smillie, 2015). (Dopaminergic neurons in the midbrain send signals reflecting reward value via axons extending to the ventral striatum and VMPFC.) In functional magnetic resonance imaging (fMRI), Extraversion has been found to predict increased neural activity in the ventral striatum in anticipation of reward (Wu, Samanez-Larkin, Katovich, 
\& Knutson, 2014), and several structural MRI studies have found that Extraversion is positively correlated with volume of VMPFC (Cremers et al., 2011; DeYoung et al., 2010; Grodin \& White, 2015; Omura, Constable, \& Canli, 2005; although other studies have not replicated this finding: Bjørnebekk et al., 2013; Hu et al., 2011; Kapogiannis et al., 2012; Liu et al., 2013).

Intelligence has been strongly linked to the cognitive control network with nodes in lateral PFC, dorsal ACC, and parietal lobes (Deary, Penke, \& Johnson, 2010; Jung \& Haier, 2007). Working memory appears to be the cognitive process most important for intelligence, and variation in the well-studied neural substrates of working memory appears to be crucial for IQ (Choi et al., 2008). Of particular relevance to the present study, one fMRI study found that neural activity in the lateral PFC during a working memory task predicted both TD and intelligence assessed outside the scanner (Shamosh et al., 2008). Further, IQ has been linked to both the functional reward response and the anatomical volume of the caudate nucleus in the striatum (Grazioplene et al., 2015; Hawes et al., 2014). The association of IQ with the caudate may reflect that Intelligence modulates how prediction-error signals in this region respond to the perceived statistical features of the environment, given that higher intelligence is likely to afford enhanced evaluation of the context of reward. Such modulation may provide a mechanism by which intelligence becomes linked to preferences through processes of basic reinforcement (Hawes et al., 2014; Chen, 2014).

Based on the clear correspondence between neural systems involved in TD and those involved in Extraversion and intelligence, the current study aimed at combining personality and fMRI data to produce a more integrated understanding of individual differences in TD. Our primary hypothesis was that Extraversion and IQ would predict distinguishable patterns of neural activity during intertemportal choice. We developed a new variation of the basic intertemporal 
choice task, in which we presented two payment options, one earlier and smaller and one later and larger, sequentially, before requiring participants to choose between them. The sequential presentation was novel and made it possible to distinguish a first phase of evaluation-when only a single option is known - from a second phase of integration - when the second and last option becomes known and during which the two options and their values can be compared. Differentiating these two phases allows a better understanding of the different contributions of the evaluation and the cognitive control systems in processing delayed rewards.

\section{Methods}

\subsection{Participants}

A sample of 304 right-handed participants between the ages of 20 and 40 years was scanned in fMRI as part of a larger study. Of these, only 250 (123 female; age: $M=26.31$ years, $S D=4.96$ ) had usable data for our analyses of the TD task; 46 were excluded due to computer or operator errors in MRI data collection; 2 were exclude for excessive movement during MRI; 4 were missing data from intelligence or personality measures; 2 were excluded for missing data in the connectivity analysis. Participants were recruited from the larger community around the twin cities of Minneapolis and St. Paul, Minnesota, using a regional classified advertisements website (Craigslist). During recruitment, potential participants were excluded for current use of psychotropic medications, including antipsychotics, anticonvulsants, and stimulants, as well as for history of neurologic or psychiatric disorders or current drug or alcohol problems. MRI contraindications (e.g., ferromagnetic implants, pacemakers) were also exclusionary. Participants were paid $\$ 50$ dollars per hour for the scanning session, plus any amount gained in the task, as well as \$20 per hour for a separate assessment session in which they completed questionnaires 
and cognitive tests. The ethical review board at the University of Minnesota approved the study.

Our target sample size (300) was chosen to enable approximately $80 \%$ power for detecting small to medium effects, even allowing some attrition and exclusion of participants whose MRI data were unusable (for example, a sample of 269 yields $80 \%$ power to detect a correlation of .17). Only about $1 / 3$ of significant effects reported in psychology are smaller than .2 , and this can therefore be considered a rough boundary between small and medium effect sizes (Hemphill, 2003). It was not until long after the study design was fixed that we encountered the article by Mahalingam et al. (2014) reporting in a very large sample that the correlation between TD and Extraversion was only about .1. In light of this finding, we were underpowered to detect this effect (power $=35 \%$ with $N=250$ ), despite our relatively large sample size by the standards of neuroimaging research; our investigation of it in this sample must therefore be considered preliminary, but given the difficulty of collecting large MRI samples, we believe it is worth reporting nonetheless.

\subsection{Procedure}

All participants completed two experimental sessions. The first session of behavioral assessments lasted 4.5-5 hours and included the personality and intelligence assessments used in the present analyses as well as other measures unrelated to the questions addressed here. Approximately two weeks later, participants underwent MRI, during which they lay supine in the scanner for approximately 1 hour and $20 \mathrm{~min}$ completing structural and functional scans. Experimental tasks were presented using E-prime 2.0 software (Psychology Software Tools, Inc.); a Sanyo projector mounted outside of the room projected through a waveguide to a screen placed in the bore of the scanner behind the subject's head, which was viewed through a mirror; 
participants entered responses via a 4-button response pad with USB interface (Current Designs, Philadelphia, PA).

\subsection{Materials}

\subsubsection{Personality}

Two questionnaires were used to assess the Big Five, the Big Five Inventory (BFI; John, Naumann, \& Soto, 2008) and the Big Five Aspect Scales (BFAS; DeYoung, Quilty, \& Peterson, 2007). Both were administered with 5-point Likert scales. The BFI uses 8-10 items to measure each of the Big Five, whereas the BFAS uses 20 items to measure each of the Big Five, with 10 items measuring each of the two major subfactors within each dimension. Composite scores for each of the Big Five were created by averaging each BFI scale with the two BFAS scales from the same dimension. Analyses involving the Big Five include only 249 participants because one was excluded for improper scale use (using only one response option or never using either of the two most extreme response options).

Intelligence was assessed using four subtests of the Wechsler Adult Intelligence Scale (Wais-IV): Block Design, Matrix Reasoning, Vocabulary, and Similarities (Wechsler, 2008). These four subtests provide a good estimate of total IQ.

\subsubsection{Intertemporal choice task}

We applied a novel design, which is a modification of a standard TD task. Two payment options - always marked by a numeric amount $x$ in \$US and a temporal delay $t$ in Days, so that an option may be completely described by a pair $(x, t)$ — were presented sequentially, for a total of 54 trials. Each trial featured one option for a smaller reward delivered sooner (Early) and one option for a larger reward delivered later (Late); among the Early options, 25 were immediate (Immediate-Early). Table 1 shows the combination of amount and delay for each trial, and 
Figure 1 shows a sequence of screen-shots of how options appeared to subjects, as well as the timeline.

This design allowed for differentiation between value-related processes that coincide with the direct comparison of two rewards (at second offer) and processes that relate to the subjective value of a single option in isolation (at first offer), which occur before the relevant information for an explicit comparison has been received. We first computed a numeric subjective value for each option, which was based on the assumption of a hyperbolic utility function for time and a fitted logit model as described in further detail below. We then considered brain activity related to the subjective value of the first option in each trial, as well as brain activity related to the subjective value of the second option on display. Because an explicit comparison of both options can take place only once the second option is known, neural signals when the first option is presented are subjective value signals produced before opportunity for explicit comparison, and those occurring in response to the second option are subjective value signals after opportunity for comparison.

For part of the sample $(N=155)$, participants always saw the Early offer first and the Late offer second (ordered presentation). This procedure resembles previous designs (e.g., Kable \& Glimcher, 2007; Ballard \& Knutson, 2009), in that the moment of the choice occurs exactly at the presentation of the Late offer. In order to eliminate this potential confound, we modified the procedure for another subsample of participants $(N=95)$, so that the presentation order of the two types of offers was randomized and both the Early and the Late offers were randomly presented as the first (First) or the second option (Second) (random presentation). Note that it was always known to the participant which type of offer was presented at each moment because the Early option was always depicted in blue and the Late option in yellow. As shown in Figure 
1, after the presentation of each offer individually, we presented participants with a third screen in which the two offers appeared together; at this point, participants indicated their choice by pressing either the blue or the yellow button on the response system, corresponding to Early or the Late offer. Having a third response step that is separated from the moment when all information has been presented serves as a potential pure control phase and aides in eliminating potential confounds of motor activation at the moment of the presentation of the second offer.

The intertemporal choice task was divided into two runs of 27 choices each and lasted on average 15 minutes. Each offer was presented for 2 seconds; the duration of the choice screen was self-paced, but was displayed for a maximum of 6 seconds, after which the words "Choose faster" were displayed. A jitter between 2 and 4 seconds was added between each screen, and between each trial.

INSERT TABLE 1 HERE

----- INSERT FIGURE 1 HERE ------

\subsection{Behavioral analysis}

We summarized subjective value with the hyperbolic TD function:

$$
U(x, t)=\frac{x}{1+k t}
$$

where the subjective value of a delayed reward, $x$, is assumed to be represented by a utility function $U(x, t)$ of the monetary reward, $x$, hyperbolically scaled according to its delay $t$ and a 
subjective parameter $k$. We assumed a soft-max choice rule for each subject in which the offer $(x$, $t)$ is chosen noisily over the offer $(y, s)$, whenever $U(x, t)$ is larger than $U(y, s)$. The noise of the softmax rule is added to the difference between $U(x, t)$ and $U(y, s)$. In summary the probability of choosing $(x, t)$ over $(y, t)$ is assumed to be:

$$
p((x, t),(y, s))=1 /(1+\exp (-\tau(U(x, t)-U(y, s)))
$$

Subjects whose behavior appeared at the boundary-that is, the 55 subjects who made all late choices (no subject made all early choices), were assigned discount rates of 0.001 , the lower bound of discount rates that could be computed from the set of choices considered in our task.

We determined each subject's subjective discount rate with the estimated parameter $\mathrm{k}$, and interpret it as a summarizing measure of bias towards sooner consumption in our task; the higher the $\mathrm{k}$, the higher a subject's tendency to be impatient for the monetary rewards offered in our experiment.

\section{5 fMRI analysis}

\subsubsection{Whole-Brain Analysis}

Image processing and statistical analysis were performed using FEAT (FMRI Expert Analysis Tool) Version 5.98, part of FSL (FMRIB's Software Library, www.fmrib.ox.ac.uk/fsl). Registration to high-resolution structural and standard space images was carried out using FLIRT (Jenkinson and Smith, 2001; Jenkinson et al., 2002). A double-gamma HRF was used for convolution; temporal derivatives were added and temporal filtering was applied. A hierarchical general linear model was applied to the data: At the within-subject level, $Z$ (Gaussianised T/F) statistic images were thresholded at $p=.05$ (uncorrected). For each subject, we included two 
regressors in a GLM, each of duration of two seconds, one for the offer presented first and one for the offer presented second. We modulated each regressor by the participant-specific subjective value of the presented offer. The moment of choice (button press) was also parameterized as a covariate in our model, but not considered an event of interest in further analysis, as it was assumed that participants made their decision once the second option was shown. This assumption is supported by average reaction times (RT) in our task being less than one second (mean RT $=783.58 \mathrm{~ms}, S D=302.57$ ) for button presses during the selection phase of the experiment.

In summary, the BOLD was estimated as follows:

$$
B O L D=\beta_{0}+\beta_{1} U(x, t)+\beta_{2} U(y, s)+\beta_{3}(U(\text { chosen })-U(\text { non }- \text { chosen }))
$$

where $\beta_{1}$ estimates the marginal effect of changes in subjective utility on changes in BOLD signal change during presentation of the first option, $\beta_{2}$ gives the same estimate for the subjective utility and presentation of the second option, and $\beta_{3}$ refers to the marginal effect of parametrically changing the difference in utility between the chosen and the un-chosen option, modeled for the period in which both options are shown side-by-side. Six regressors of no interest modeled head motion. Higher-level analysis, across subjects, was carried out using FLAME (Beckmann et al., 2003; Woolrich et al., 2004; Woolrich, 2008). At this second level, Z (Gaussianised T/F) statistic images were thresholded using clusters determined by Z > $3.2(p<$ $.001)$ and a (corrected) cluster significance threshold of $p=.05$ (Worsley, 2001).

\subsubsection{Time-series analysis}

Time-series were extracted for each participant from clusters of 27 voxels each, created 
with fslmaths (FMRIB's Software Library), centered at the activation peak of the larger clusters resulting from the whole-brain analysis at the moment of presentation of both the first and the second option. We extracted the mean BOLD path in each cluster and regressed it in a panel analysis on both trait variables and trial specific variables (such as subjective values of offers). The panel data analysis on the BOLD path is a more flexible tool that allows us to study the effect of individual variables (such as IQ) and their interactions (such as that between IQ and subjective value).

\subsubsection{Functional connectivity}

In order to run the beta-series connectivity analysis, a new model was run in $\mathrm{AFNI}^{1}$ (Cox, 1996), in which a single-trial beta (two beta coefficients for each trial, one corresponding to the first and one to the second offer) was computed in a whole-brain analysis. Each offer (first and second) was considered an event of interest, so that 108 parameters functioned as separate regressors in an initial GLM that included also one regressor to model the average response to the choice screen, and 6 regressors of no interest to model head motion. Subjective value was not used to modulate the offers in this analysis. The resulting 108 parameter estimates were collected as a single series of beta-values for each subject, which then provided the basis for a functional connectivity analysis as described in (Rissman et al., 2004). The assumption of this method is that the regions whose beta-series are correlated during a certain event of interest (for example, the second offer) are considered to be functionally interacting for that event (see Rissman et al., 2004, p. 755 for the test of the validity of the assumption).

The anatomically defined VMPFC was used as the seed region for our functional connectivity analysis. This area was chosen because the whole-brain analysis revealed its significant activation at the display of the second offer, confirming its well-known role in 
representing decision-values (Levy and Glimcher, 2012), and because its volume has been shown repeatedly to be associated with Extraversion.

We proceeded to consider individual differences in strength of connectivity. For each subject we computed separately the beta-series estimates only for the regions functionally connected to the VMPFC at the group-level, and we performed a correlation between VMPFC beta-series and the extracted beta-series from these regions. The parameter of correlation (Pearson's $r$, Fisher-transformed) measures the subject-specific strength of correlation, or strength of functional connectivity, between VMPFC and each area.

\section{Results}

\subsection{Behavioral results}

Our design allows identification of a hyperbolic discount factor ( $\mathrm{k}$ in equation (1)) larger than $\mathrm{k}_{\min }=0.0071$. 55 of our subjects chose the option with a late payment in all trials, so they have a $\mathrm{k}$ value smaller than $\mathrm{k}_{\mathrm{min}}$. Note that the variability on the estimated subjective value for subjects with $\mathrm{k}$ in the interval is for any option less than 13 percent, with an average of 6 percent. The estimated discount values $(k)$ provide a good summary of preferences and predict behavior reasonably well in our task; for example, the $R^{2}$ of the regression of the fraction of choice of late options on the estimated discount factor is .76. The respective distributions for $k$ did not differ between groups that saw a random (early and late offers randomly presented as the first or the second option in the sequence) versus ordered (early and late offers always presented as first and second options in the sequence, respectively) presentation of rewards (ordered: $M=-1.72, S D=$ 0.96; random: $\left.M=-1.84, S D=0.68 ; t_{(248)}=-1.198, p=.232\right)$.

Extraversion and IQ were correlated with the $\log$ of $k$ in a manner consistent with past 
results (E: Spearman's rho $=.11, p=.092$; IQ: rho $=-.32, p<.001)$. (Nonparametric correlations were used because $k$ values were not normally distributed even after log transformation, due to the 55 participants who always chose the late option.) Note that although the association with Extraversion did not reach significance at $p<.05$, it was almost exactly the same magnitude as reported in a very large sample by Mahalingam et al. (2014). Correlations with the other Big Five traits were weaker; $\mathrm{N}=.04, \mathrm{C}=.06, \mathrm{O}=-.08, \mathrm{~A}:-.07$. Given that our neural hypotheses involved only Extraversion and IQ, we did not analyze the other Big Five traits in further analyses.

\section{2 fMRI results}

\subsubsection{Whole-brain analysis}

We identified neural correlates of subjective value $U(x, t)$ (see Methods) for each offer being presented. Figure $2 \mathrm{a}$ and Table 2 show that activation during the presentation of the first offer was proportional to the estimated subjective value of the first offer within a large cluster extending from right middle to right inferior frontal gyrus (right DLPFC extending to ventrolateral PFC), a smaller cluster in the left middle frontal gyrus (left DLPFC), clusters in the mid-paracingulate gyrus, bilateral anterior insula (AI) and bilateral striatum (caudate). Figure $2 \mathrm{~b}$ and Table 3 show that positive correlations with subjective value of the second offer, during its presentation, were present in the VMPFC, dACC, posterior cingulate (PCC), bilateral striatum, and bilateral anterior middle frontal gyrus (DLPFC).

We found significant differences in modulated brain activity, depending on whether an offer was shown first (isolated evaluation phase) or second (integration phase). The contrast of parametrically modulated activation responses revealed a significantly more pronounced response to subjective value for VMPFC and parts of the caudate during integration (Figure 2c 
and Table 4a). The reverse contrast showed greater activation within a small region of the left superior parietal lobe during isolated evaluation, suggesting greater importance of this region for choice preparation pre-integration, before the alternative choice object becomes available (Figure $2 d$ and Table $4 b)$.

No differences in activation were found for ordered versus random presentation, suggesting that our results are related to the sequential presentation of the options, and not to whether a sooner or a later option is shown first. This result is mentioned in particular because subjects knew whether an offer was early or late for a given choice in our design, so that first options in the random presentation order should not have been treated by default as early. ${ }^{2}$

----- INSERT TABLES 2, 3, 4 HERE ------

----- INSERT FIGURE 2 HERE ------

\subsubsection{Functional connectivity}

Contrasts of parameters from the Rissman method (see Methods section) of functional connectivity with VMPFC as a seed showed significantly greater functional connectivity during presentation of the second offer for multiple connected brain regions. After correction for spatial correlation using a Monte Carlo simulation-based estimator ${ }^{3}$ (AFNI 3dClustSim (Forman et al., 1995; Ward, 2000)), clusters showing stronger functional connectivity to VMPFC during the presentation of the second offer (contrasted with the first) corresponded anatomically to the left middle frontal gyrus (DLPFC) (BA 46 and BA 6), left insula, right parietal lobe (peaking at the superior parietal lobule (SPL)), left inferior parietal lobule, and mid-cingulate gyrus (mACC) (Figure 3, Table 5). No region was identified as more functionally connected to VMPFC during presentation of the first offer with respect to the second. 
----- INSERT TABLE 5 HERE ------

----- INSERT FIGURE 3 HERE ------

\subsection{Correlations with personality of neural activity and connectivity}

\subsubsection{Neural activity (time-series analysis)}

We analyzed the correlation of IQ and Extraversion with activation corresponding to the evaluation of the subjective value of the two offers in a set of 8 anatomical regions. From each region we selected a smaller cluster of 27 voxels in a cube of $9 \mathrm{~mm}$ per side, centered at the peak activation in that region.

IQ was significantly correlated with neural response to the subjective values of offers in the bilateral caudate. In particular we found that the response to the subjective value of the first offer (SVO1) could be decomposed into a positive coefficient for subjective value (standardized coefficient $\beta=.02, p<.001)$, a negative coefficient for IQ $(\beta=-.36, p=.001)$ and a negative interaction with SVO1 $(\beta=-.011, p=.013$; the ratio between the coefficients of interaction and IQ is 0.03). All coefficients, except that for the interaction between SVO1 and IQ, are significantly different from zero at the 5 per cent level after correction for multiple comparisons. Similar responses, but weaker, are found in the VMPFC $(\beta=.01, p<.001$ for SVO1, $\beta=-.16, p$ .09 for IQ, $\beta=-.007, p=.066$ for interaction between IQ and SVO1). Extraversion did not predict activation in any cluster.

\subsubsection{Functional connectivity}

Five regions were identified by significant functional connectivity to VMPFC at the presentation of the second offer; thus, we examined the strength of correlations of the five connectivity variables characterizing the strength of these functional connections with 
Extraversion and IQ. Three of the five regions showed correlations with Extraversion in the range of .10-.15 (with $p$-values ranging from .035 to .059). Correlations with IQ were weaker, with only one approaching significance $(r=-.11, p=.071)$. Because the five connectivity variables were highly intercorrelated, we performed a principal components analysis, which was justified in view of the large squared multiple correlation of the variables (ranging from .68 to .84; Kaiser-Meyer-Olkin measure of sampling adequacy: 0.74). The eigenvalues clearly indicated a single general factor (eigenvalues $=3.53,0.89,0.30,0.18,0.08$ ), which explained $70.6 \%$ of the variance, with loadings ranging from .77-.90. We, therefore, created a composite index of VMPFC connectivity by averaging across the 5 regions, and this score was positively correlated with Extraversion, $r=.13, p=.039$. The correlation with IQ was $-.07, p=.229$.

\section{Discussion}

Intelligence and Extraversion are known to predict TD, and this study tested the hypothesis that these two traits would be linked to the neural underpinnings of intertemporal choice, based on parallels between what is known about the neural correlates of TD and the neural correlates of IQ and Extraversion. In our analysis of behavior, we found an association of higher intelligence with greater patience (reduced TD), confirming well-established findings (Shamosh \& Gray, 2008; Burks et al., 2009). We additionally found a positive correlation between greater Extraversion and impatience (increased TD) of the same magnitude reported in a sample of 5,888 (Mahalingam et al., 2014), although it did not quite reach significance given our sample size. Despite being underpowered to study this Extraversion effect, we decided to examine its neural correlates to provide at least a preliminary evaluation of that component of our hypothesis. 
We focused on subjective value as our behaviorally elicited index of TD and showed that subjective value signals were present in the brain for each option as they were shown to participants. Signals corresponding to subjective value were stronger in VMPFC when comparison between options occurred, and were substantially weaker in our analysis during presentation of the first option before such explicit comparison could take place. This result suggests that the process of explicit comparison contributes distinctly to the unified subjective value signals that guide decision in VMPFC, when (as in our design) evaluation and comparison can be separately identified. This does not rule out, however, that such value signals may also be present in the absence of explicit comparison.

Subjective value signals in the ventral striatum were similarly present during presentation of both the first and second option (differently from VMPFC, where activation was significantly stronger during presentation of the second option). This is consistent with the literature concerning the ventral striatum's association with anticipatory reward responses in proportion to their expected magnitude (Knutson \& Peterson, 2005) and with subjective value in general (Kable \& Glimcher, 2007). Intelligence was negatively associated with modulated activation of the bilateral caudate, a finding that is consistent with previous results linking intelligence to neural response to rewards in the caudate (Hawes et al., 2014) and to the volume of this subcortical structure (Grazioplene et al., 2015). Our results are consistent with the idea that intelligence contributes to modulation of prediction error signals in the caudate, and that these modulatory effects impact value representation during decision, perhaps by assisting adaptive representation of reward values in context versus in isolation (i.e. representation of the relevant properties of the environment) (Rustichini, 2015). Assuming such an adaptation mechanism contributes indiscriminately to reward representation during evaluation of delayed rewards and 
probabilistic rewards, this explanation may extend to observed correlations of IQ with impulsivity and risk aversion also (Rustichini, 2009; Burks et al, 2009).

The analysis of functional connectivity revealed a network of regions functionally connected to the VMPFC during the offer comparison phase; the network encompassed regions of the left DLPFC, left insula, ACC, and bilateral parietal lobule. The identified region in the DLPFC encompasses areas that have been considered functionally critical to self-control (see Hare et al, 2009, although the peak of our activation is a bit more anterior), and the fact that the connectivity between VMPFC and left DLPFC was stronger when participants were presented with the second option relative to the first suggests that the involvement of DLPFC for intertemporal choice behavior was specific to the comparison of rewards. This result is consistent with previous findings identifying the functional circuit encompassing VMPFC and DLPFC as crucial for the last stage of the decision-making process (Hare et al., 2009; Hare, Malmaud, \& Rangel, 2011; Hare et al., 2014; Figner et al., 2010). The activations of insula, ACC and inferior parietal lobe have also been found associated with evaluation of rewarding stimuli (Paulus \& Frank, 2003), and their connectivity with VMPFC during the comparison phase presumably represents their contribution to the value computation.

We found that Extraversion positively predicted the strength of this functional network, consistent with previous findings linking Extraversion to anatomical variation in VMPFC (Cremers et al., 2011; DeYoung et al., 2010; Grodin \& White, 2015; Omura et al., 2005) and theories of Extraversion that link it to sensitivity to reward (e.g., Depue \& Collins, 1999; DeYoung, 2015). Nonetheless, this association was weak, and our study was not well powered to detect it. Future research on this effect should use a larger sample.

\subsection{Conclusions}


The results reported in this paper identify relations between behavior in delaydiscounting choices, neural substrates of reward evaluation, and individual differences in personality, and they suggest avenues for their further investigation. They suggest that understanding caudate function will be particularly important for understanding the association of intelligence with TD. They also suggest that the association between Extraversion and TD is linked to the role that a network centered on VMPFC plays in evaluating rewards. More broadly, these results are relevant for psychological theory, as they move us toward an integrated understanding of the neural basis of individual differences: personality theory offers a theoretical frame in which individual differences in decision-making can be interpreted, while decision theory offers formal models of the outcome (the choice) of a process that is not modeled. The integration of these theories may lead to an understanding of the fundamental links between personality traits and economic preferences, thereby allowing, for example, a better prediction of economic and personal success (Rustichini, DeYoung, Anderson, \& Burks, 2012). The inclusion of neuroscientific data is crucial in order to understand the mechanisms underlying the overt choice, such as reward processing and choice evaluation, known to be correlated with specific personality profiles. Our findings support the importance of reward and prediction-error signals for coordinating the representation of value, in particular during direct comparison of rewards, while additionally illustrating the relevance of individual differences to understanding variation in this functional mechanism. 
Personality and delay discounting in fMRI 23

\section{ACKNOWLEDGMENTS}

This study was supported by a grant to Colin DeYoung from the National Institute on Drug Abuse (NIDA; R03 DA029177-01A1) and to Colin DeYoung and Aldo Rustichini from the National Science Foundation (SES-1061817). We thank Matt Paffel and Rachael Grazioplene for their help with subject recruitment and data collection. 


\section{Reference}

1. Ballard, K., \& Knutson, B. (2009). Dissociable neural representations of future reward magnitude and delay during temporal discounting. Neuroimage, 45(1), 143-150.

2. Beckmann, C. F., Jenkinson, M., \& Smith, S. M. (2003). General multilevel linear modeling for group analysis in FMRI. Neuroimage, 20(2), 1052-1063.

3. Bjørnebekk, A., Fjell, A. M., Walhovd, K. B., Grydeland, H., Torgersen, S., \& Westlye, L. T. (2013). Neuronal correlates of the five factor model (FFM) of human personality: Multimodal imaging in a large healthy sample. NeuroImage, 65, 194-208.

4. Burks, S. V., Carpenter, J. P., Goette, L., \& Rustichini, A. (2009). Cognitive skills affect economic preferences, strategic behavior, and job attachment. Proceedings of the National Academy of Sciences, 106(19), 7745-7750.

5. Chen, C. (2014). Intelligence moderates reinforcement learning: a mini-review of the neural evidence. Journal of neurophysiology, jn-00600.

6. Choi, Y. Y., Shamosh, N. A., Cho, S. H., DeYoung, C. G., Lee, M. J., Lee, J. M., ... \& Lee, K. H. (2008). Multiple bases of human intelligence revealed by cortical thickness and neural activation. The journal of neuroscience, 28(41), 10323-10329.

7. Cox, R.W. (1996). AFNI: Software for Analysis and Visualization of Functional Magnetic Resonance Neuroimages. Computers and Biomedical Research 29, 162-173.

8. Cremers, H., van Tol, M. J., Roelofs, K., Aleman, A., Zitman, F. G., van Buchem, M. A., Veltman, D. J., \& van der Wee, N. J. (2011). Extraversion is linked to volume of the orbitofrontal cortex and amygdala. PloS one, 6(12), e28421.

9. Deary, I. J., Penke, L., \& Johnson, W. (2010). The neuroscience of human intelligence differences. Nature Reviews Neuroscience, 11(3), 201-211. 
10. Depue, R. A., \& Collins, P. F. (1999). Neurobiology of the structure of personality: Dopamine, facilitation of incentive motivation, and extraversion. Behavioral and Brain Sciences, 22(03), 491-517.

11. Depue, R. A., \& Fu, Y. (2013). On the nature of extraversion: variation in conditioned contextual activation of dopamine-facilitated affective, cognitive, and motor processes. Frontiers in human neuroscience, 7.

12. DeYoung, C. G. (2013). The neuromodulator of exploration: A unifying theory of the role of dopamine in personality. Frontiers in human neuroscience, 7.

13. DeYoung, C. G. (2015). Cybernetic big five theory. Journal of Research in Personality, $56,33-58$.

14. DeYoung, C.G., Quilty, L.C., \& Peterson, J.B. (2007). Between facets and domains: 10 aspects of the Big Five. Journal of Personality and Social Psychology 93, 880-896.

15. DeYoung, C.G., Hirsh, J.B., Shane, M.S., Papademetris, X., Rajeevan, N., \& Gray, J.R. (2010). Testing predictions from personality neuroscience: Brain structure and the Big Five. Psychological Science, 21, 820-828.

16. Duckworth, A.L., \& Seligman, M.E.P. (2005). Self-discipline outdoes IQ in predicting academic performance of adolescents. Psychological Science, 16, 939-944.

17. Duckworth, A. L., Tsukayama, E., \& Kirby, T. A. (2013). Is it really self-control? Examining the predictive power of the delay of gratification task. Personality and Social Psychology Bulletin, 0146167213482589.

18. Evans, G.W., \& Rosenbaum, J. (2008). Self-regulation and the income-achievement gap. Early Childhood Research Quarterly 23, 504-514.

19. Figner, B., Knoch, D., Johnson, E.J., Krosch, A.R., Lisanby, S.H., Fehr, E., \& Weber, 
E.U. (2010). Lateral prefrontal cortex and self-control in intertemporal choice. Nature Neuroscience 13, 538-539.

20. Forman, S. D., Cohen, J. D., Fitzgerald, M., Eddy, W. F., Mintun, M. A., \& Noll, D. C. (1995). Improved assessment of significant activation in functional magnetic resonance imaging (fMRI): use of a cluster-size threshold. Magnetic Resonance in medicine, 33(5), 636-647.

21. Frederick, S., Loewenstein, G., \& O'donoghue, T. (2002). Time discounting and time preference: A critical review. Journal of economic literature, 351-401.

22. Grazioplene, R.G., G. Ryman, S., Gray, J.R., Rustichini, A., Jung, R.E., and DeYoung, C.G. (2015). Subcortical intelligence: Caudate volume predicts IQ in healthy adults. Human Brain Mapping, 36, 1407-1416.

23. Gray, J. A., \& McNaughton, N. (2000). The neuropsychology of anxiety: An enquiry into the function of the septo-hippocampal system. New York: Oxford University Press.

24. Grodin, E. N., \& White, T. L. (2015). The neuroanatomical delineation of agentic and affiliative extraversion. Cognitive, Affective, \& Behavioral Neuroscience, 15(2), 321-334.

25. Hare, T.A., Camerer, C.F., \& Rangel, A. (2009). Self-Control in decision-making Involves modulation of the vmPFC valuation system. Science 324, 646-648.

26. Hare, T.A., Malmaud, J., \& Rangel, A. (2011). Focusing attention on the health aspects of foods changes value signals in vmPFC and improves dietary choice. Journal of Neuroscience. 31, 11077-11087.

27. Hare, T.A., Hakimi, S., \& Rangel, A. (2014). Activity in dlPFC and its effective connectivity to vmPFC are associated with temporal discounting. Frontiers in Neuroscience, 8 . 
28. Hawes, D.R., DeYoung, C.G., Gray, J.R., \& Rustichini, A. (2014). Intelligence moderates neural responses to monetary reward and punishment. Journal of Neurophysiology 111, $1823-1832$.

29. Hirsh, J.B., Morisano, D., \& Peterson, J.B. (2008). Delay discounting: Interactions between personality and cognitive ability. Journal of Research in Personality 42, 16461650.

30. Hirsh, J. B., \& Inzlicht, M. (2008). The devil you know neuroticism predicts neural response to uncertainty. Psychological Science, 19(10), 962-967.

31. Hirsh, J. B., Guindon, A., Morisano, D., \& Peterson, J. B. (2010). Positive mood effects on delay discounting. Emotion, 10(5), 717.

32. Hu, X., Erb, M., Ackermann, H., Martin, J. A., Grodd, W., \& Reiterer, S. M. (2011). Voxel-based morphometry studies of personality: Issue of statistical model specificationeffect of nuisance covariates. NeuroImage, 54(3), 1994-2005.

33. Jenkinson, M., \& Smith, S. (2001). A global optimisation method for robust affine registration of brain images. Medical image analysis, 5(2), 143-156.

34. Jenkinson, M., Bannister, P., Brady, M., \& Smith, S. (2002). Improved optimization for the robust and accurate linear registration and motion correction of brain images. Neuroimage, 17(2), 825-841.

35. John, O. P., Naumann, L. P., \& Soto, C. J. (2008). Paradigm shift to the integrative Big Five trait taxonomy. Handbook of personality: Theory and research, 3, 114-158.

36. Jung, R. E., \& Haier, R. J. (2007). The Parieto-Frontal Integration Theory (P-FIT) of intelligence: converging neuroimaging evidence. Behavioral and Brain Sciences, 30(02), $135-154$. 
37. Liu, W.-Y., Weber, B., Reuter, M., Markett, S., Chu, W.-C., \& Montag, C. (2013). The big five of personality and structural imaging revisited: A VBM-DARTEL study. Neuroreport, 24(7), 375-380.

38. Kable, J.W., \& Glimcher, P.W. (2007). The neural correlates of subjective value during intertemporal choice. Nature Neuroscience 10, 1625-1633.

39. Kable, J.W., \& Glimcher, P.W. (2009). The Neurobiology of Decision: Consensus and Controversy. Neuron 63, 733-745.

40. Kapogiannis, D., Sutin, A., Davatzikos, C., Costa, P., \& Resnick, S. (2013). The five factors of personality and regional cortical variability in the Baltimore longitudinal study of aging. Human Brain Mapping, 34(11), 2829-2840.

41. Knutson, B., \& Peterson, R. (2005). Neurally reconstructing expected utility. Games and Economic Behavior 52, 305-315.

42. Krueger, R.F., Caspi, A., Moffitt, T.E., Silva, P.A., \& McGee, R. (1996). Personality traits are differentially linked to mental disorders: A multitrait-multidiagnosis study of an adolescent birth cohort. Journal of Abnormal Psychology 105, 299-312.

43. Levy, D. J., \& Glimcher, P. W. (2012). The root of all value: a neural common currency for choice. Current opinion in neurobiology, 22(6), 1027-1038.

44. Mahalingam, V., Stillwell, D., Kosinski, M., Rust, J., \& Kogan, A. (2014). Who can wait for the future? A personality perspective. Social Psychological and Personality Science, $5(5), 573-583$.

45. McClure, S.M., Laibson, D.I., Loewenstein, G., \& Cohen, J.D. (2004). Separate neural systems value immediate and delayed monetary rewards. Science 306, 503-507.

46. Omura, K., Constable, R. T., \& Canli, T. (2005). Amygdala gray matter concentration is 
associated with Extraversion and neuroticism. Neuroreport, 16(17), 1905-1908

47. Ostaszewski, P. (1996). The relation between temperament and rate of temporal discounting. Eur. J. Pers. 10, 161-172.

48. Peters, J., \& Büchel, C. (2011). The neural mechanisms of inter-temporal decisionmaking: understanding variability. Trends in Cognitive Sciences 15, 227-239.

49. Rangel, A., \& Clithero, J. A. (2013). The computation of stimulus values in simple choice. Neuroeconomics: Decision making and the brain, 2.

50. Rissman, J., Gazzaley, A., \& D’Esposito, M. (2004). Measuring functional connectivity during distinct stages of a cognitive task. NeuroImage 23, 752-763.

51. Rustichini, A. (2009). Neuroeconomics: what have we found, and what should we search for. Current opinion in neurobiology, 19(6), 672-677

52. Rustichini, A. (2015). The role of intelligence in economic decision-making. Current Opinion in Behavioral Sciences, 5, 32-36.

53. Rustichini, A., DeYoung, C. G., Anderson, J. E., \& Burks, S. V. (2012). Toward the Integration of Personality Theory and Decision Theory in the Explanation of Economic and Health Behavior (No. 6750). Institute for the Study of Labor (IZA). Chicago

54. Shamosh, N.A., \& Gray, J.R. (2008). Delay discounting and intelligence: A metaanalysis. Intelligence 36, 289-305.

55. Shamosh, N. A., DeYoung, C. G., Green, A. E., Reis, D. L., Johnson, M. R., Conway, A. R., .. \& Gray, J. R. (2008). Individual differences in delay discounting relation to intelligence, working memory, and anterior prefrontal cortex. Psychological Science, 19(9), 904-911.

56. Verdejo-García, A., Lawrence, A.J., \& Clark, L. (2008). Impulsivity as a vulnerability 
marker for substance-use disorders: Review of findings from high-risk research, problem gamblers and genetic association studies. Neuroscience \& Biobehavioral Reviews 32, $777-810$.

57. Wacker, J., \& Smillie, L. D. (2015). Trait Extraversion and Dopamine Function. Social and Personality Psychology Compass, 9(6), 225-238.

58. Ward, B. D. (2000). Simultaneous inference for fMRI data. AFNI 3dDeconvolve Documentation, Medical College of Wisconsin.

59. Wechsler, D. (2008). Wechsler adult intelligence scale-Fourth Edition (WAIS-IV). San Antonio, TX: NCS Pearson.

60. Woo, C. W., Krishnan, A., \& Wager, T. D. (2014). Cluster-extent based thresholding in fMRI analyses: pitfalls and recommendations. Neuroimage, 91, 412-419.

61. Woolrich, M. W., Behrens, T. E., Beckmann, C. F., Jenkinson, M., \& Smith, S. M. (2004). Multilevel linear modelling for FMRI group analysis using Bayesian inference. Neuroimage, 21(4), 1732-1747.

62. Woolrich, M. (2008). Robust group analysis using outlier inference. Neuroimage, 41(2), 286-301.

63. Worsley, K. J. (2001). Statistical analysis of activation images. Functional MRI: An introduction to methods, $14,251-70$.

64. Wu, C. C., Samanez-Larkin, G. R., Katovich, K., \& Knutson, B. (2014). Affective traits link to reliable neural markers of incentive anticipation. NeuroImage, 84, 279-289. 


\section{Footnotes}

1. The change in software was due to relative convenience of the process for dealing with beta-series for the functional connectivity analysis in AFNI as compared to FSL.

2. In-depth analysis and interpretation of the sequential choice paradigm, focusing on general decisionmaking processes rather than individual differences, will be reported as a separate study, as the scope of the current report is to discuss the relation of personality to the neural underpinnings of TD.

3. A family-wise error (FWE) correction at $p<.05$ was achieved with a cluster-defining threshold of two-tailed $p<.0007$ and a cluster size of at least 83 voxels. The small $\mathrm{p}$-value was chosen in light of the high power of the study (Woo, Krishnan, \& Wager, 2014). 


\section{Figure Captions}

Figure 1. Example of trial. The first offer is presented for 2 seconds, then, after a 2 or 4 seconds jitter, the second offer appears for another 2 seconds; after a second jitter, a screen depicting both offers is shown, and participants have to choose between the blue and the yellow option. In the fixed presentation, the early offer is always presented first; in both presentation orders, however, the early option is always depicted in blue, and the late option in yellow. For the fMRI analysis, we focused on the first two time windows (offer 1 and offer 2).

Figure 2. Activations from whole-brain analysis. Areas that increase their activation with the subjective value of A) offer 1 and of B) offer 2, at the moment of presentation of the stimuli. The lower part of the figure shows the areas that increase their activation more for the subjective value of offer 2 compared to offer 1 (C) ) and vice-versa (D) ).

Figure 3. Activations from functional connectivity analysis. Areas whose functional connectivity with VMPFC is higher at the presentation of the second offer compared to the first offer. 


\begin{tabular}{|c|c|}
\hline \multicolumn{2}{|c|}{ Stimuli } \\
\hline Early option & Late option \\
\hline$\$ 10$ Today & $\$ 19$ in 14 days \\
\hline$\$ 15$ Today & $\$ 21$ in 7 days \\
\hline$\$ 20$ Today & $\$ 30$ in 7 days \\
\hline$\$ 10$ Today & $\$ 22$ in 7 days \\
\hline$\$ 20$ in 7 days & $\$ 25$ in 21 days \\
\hline$\$ 10$ in 7 days & $\$ 12$ in 14 days \\
\hline$\$ 20$ Today & $\$ 28$ in 14 days \\
\hline$\$ 15$ in 14 days & $\$ 25$ in 21 days \\
\hline$\$ 20$ in 7 days & $\$ 21$ in 14 days \\
\hline$\$ 15$ in 7 days & $\$ 20$ in 14 days \\
\hline$\$ 15$ in 14 days & $\$ 30$ in 21 days \\
\hline$\$ 20$ in 7 days & $\$ 30$ in 21 days \\
\hline$\$ 20$ Today & $\$ 30$ in 14 days \\
\hline$\$ 15$ Today & $\$ 25$ in 14 days \\
\hline$\$ 20$ in 14 days & $\$ 35$ in 21 days \\
\hline$\$ 10$ in 14 days & $\$ 12$ in 21 days \\
\hline$\$ 20$ Today & $\$ 30$ in 21 days \\
\hline$\$ 15$ Today & $\$ 28$ in 21 days \\
\hline$\$ 15$ Today & $\$ 25$ in 7 days \\
\hline$\$ 10$ Today & $\$ 24$ in 21 days \\
\hline$\$ 15$ Today & $\$ 30$ in 14 days \\
\hline$\$ 15$ in 7 days & $\$ 30$ in 21 days \\
\hline$\$ 20$ in 7 days & $\$ 25$ in 14 days \\
\hline$\$ 20$ in 7 days & $\$ 28$ in 21 days \\
\hline$\$ 15$ Today & $\$ 25$ in 14 days \\
\hline$\$ 10$ in 7 days & $\$ 24$ in 21 days \\
\hline$\$ 10$ in 7 days & $\$ 28$ in 21 days \\
\hline$\$ 10$ in 7 days & $\$ 12$ in 21 days \\
\hline$\$ 10$ Today & $\$ 11$ in 14 days \\
\hline$\$ 15$ in 7 days & $\$ 30$ in 14 days \\
\hline$\$ 20$ in 7 days & $\$ 30$ in 14 days \\
\hline$\$ 10$ in 14 days & $\$ 20$ in 21 days \\
\hline
\end{tabular}

Table 1. Task stimuli: combination of amount and delay. 


\begin{tabular}{|c|c|c|c|c|c|c|}
\hline \multirow[b]{2}{*}{ Local maxima } & \multicolumn{4}{|c|}{ First offer (SV modulated) } & \multirow[b]{2}{*}{$\mathbf{y}$} & \multirow[b]{2}{*}{$\mathbf{z}$} \\
\hline & Voxels & $\mathbf{P}$ & Z-MAX & $\mathbf{x}$ & & \\
\hline & 13477 & 0.0001 & & & & \\
\hline R Occipital Pole & & & 7.17 & 16 & -96 & 10 \\
\hline L Occipital Pole & & & 6.4 & -8 & -100 & 12 \\
\hline L Lingual Gyrus & & & 6.01 & -16 & -66 & -2 \\
\hline L Intracalcarine cortex & & & 5.96 & -10 & -76 & 6 \\
\hline \multirow[t]{2}{*}{ R Precuneus } & & & 5.95 & 18 & -68 & 42 \\
\hline & 7969 & 0.0001 & & & & \\
\hline R Ant Insula/OFC & & & 7.11 & 34 & 22 & -8 \\
\hline Paracingulate Gyrus & & & 6.58 & 8 & 30 & 42 \\
\hline R Inferior Frontal Gyrus & & & 6.19 & 52 & 20 & 26 \\
\hline R Middle Frontal Gyrus & & & 6.18 & 30 & 8 & 56 \\
\hline R Middle Frontal Gyrus & & & 6.06 & 54 & 30 & 24 \\
\hline \multirow[t]{2}{*}{ Paracingulate/ACC } & & & 5.11 & -4 & 34 & 30 \\
\hline & 619 & 0.00564 & & & & \\
\hline \multirow[t]{2}{*}{ L Ant Insula } & & & 6.81 & -32 & 18 & -6 \\
\hline & 573 & 0.00765 & & & & \\
\hline L Inferior Frontal Gyrus & & & 4.26 & -46 & 20 & 18 \\
\hline L Middle Front Gyrus & & & 4.18 & -48 & 20 & 30 \\
\hline \multirow[t]{2}{*}{ L Middle Front Gyrus } & & & 3.51 & -48 & 10 & 34 \\
\hline & 422 & 0.0221 & & & & \\
\hline R Caudate & & & 5.55 & 12 & 12 & 4 \\
\hline
\end{tabular}

Table 2. Areas whose activation survived FSL cluster correction $(z=3.2, p<0.001)$ at the moment of the presentation of the first offer. The coordinates are anatomical MNI, and the areas have been identified with FSL Harvard-Oxford atlas. 


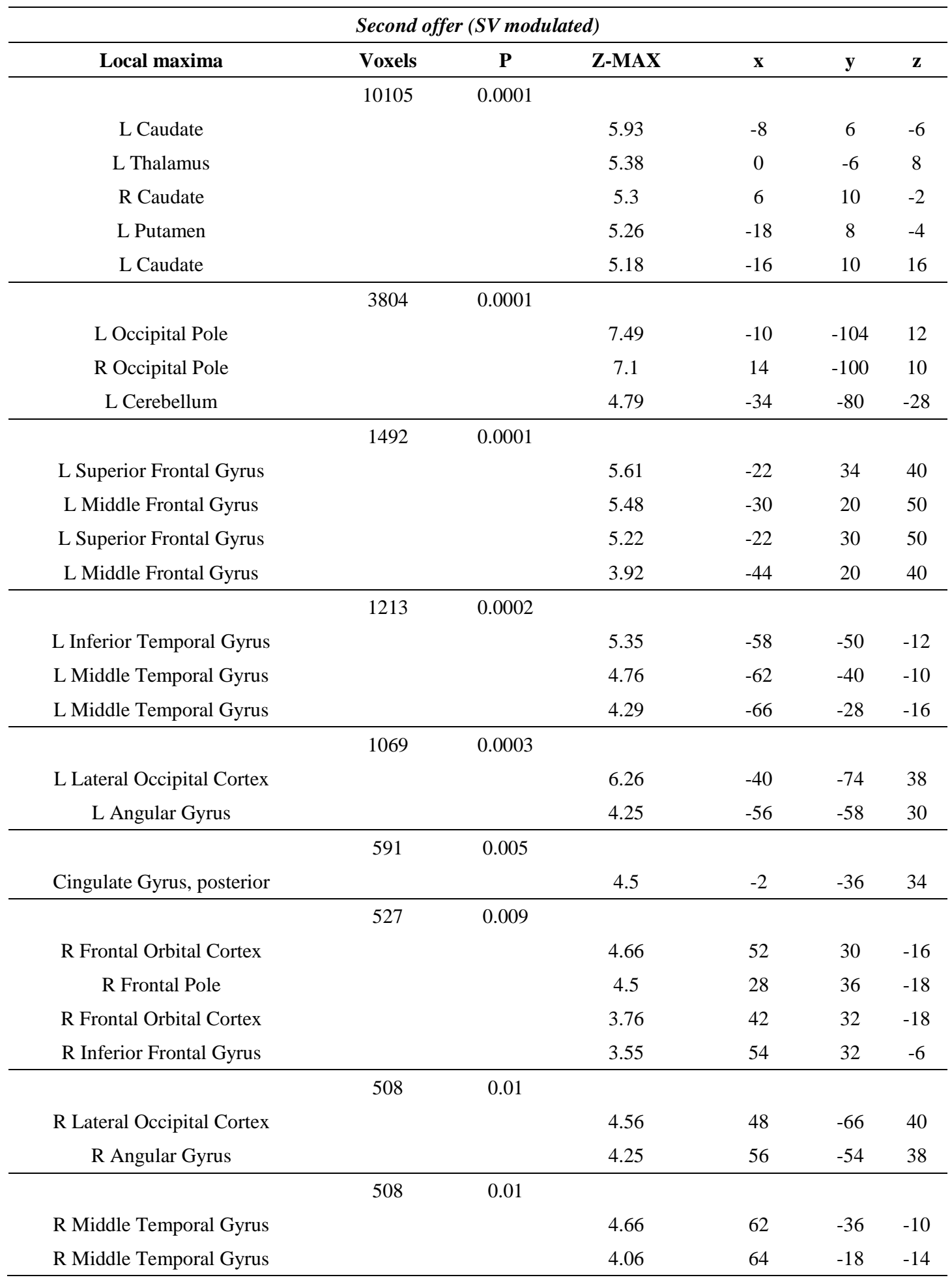

Table 3. Areas whose activation survived FSL cluster correction $(z=3.2, p<0.001)$ at the moment of the presentation of the second offer. The coordinates are anatomical MNI, and the areas have been identified with FSL Harvard-Oxford atlas. 


\begin{tabular}{|c|c|c|c|c|c|c|}
\hline \multicolumn{7}{|c|}{ a) Second offer-First Offer } \\
\hline Local maxima & Voxels & $\mathbf{P}$ & Z-MAX & $\mathbf{x}$ & $\mathbf{y}$ & $\mathbf{z}$ \\
\hline & 1527 & $<0.0001$ & & & & \\
\hline L Caudate & & & 5.2 & -18 & 8 & 18 \\
\hline L Caudate & & & 5.16 & -20 & -4 & 22 \\
\hline L Frontal Pole & & & 4.72 & -20 & -36 & -8 \\
\hline L Frontal Medial Cortex & & & 4.1 & -16 & 32 & -20 \\
\hline \multirow[t]{2}{*}{ L Putamen } & & & 3.6 & -22 & -2 & 12 \\
\hline & 885 & 0.0001 & & & & \\
\hline R Caudate & & & 5.38 & 20 & 2 & 22 \\
\hline R Caudate & & & 4.86 & 20 & 22 & 12 \\
\hline \multirow[t]{2}{*}{ R Caudate } & & & 4.54 & 18 & 28 & -6 \\
\hline & 465 & 0.01 & & & & \\
\hline R Occipital Gyrus & & & 4.49 & 6 & -84 & -24 \\
\hline R Occipital Gyrus & & & 4.36 & 10 & -92 & -18 \\
\hline L Lingual Gyrus & & & 4.02 & -8 & -88 & -24 \\
\hline \multicolumn{7}{|c|}{ b) First Offer - Second Offer } \\
\hline & 6791 & $<0.0001$ & & & & \\
\hline R Intracalcarine Cortex & & & 6.31 & 14 & -72 & 16 \\
\hline R Intracalcarine Cortex & & & 5.84 & 20 & -70 & 10 \\
\hline R Cuneal Cortex & & & 5.75 & 18 & -76 & 36 \\
\hline L Lingual Gyrus & & & 5.67 & -18 & -68 & -2 \\
\hline \multirow{2}{*}{ L Intracalcarine Cortex } & & & 5.58 & -14 & -70 & 12 \\
\hline & 583 & 0.005 & & & & \\
\hline R Occipital Gyrus & & & 4.57 & -46 & -20 & 48 \\
\hline R Occipital Gyrus & & & 4.26 & -32 & -32 & 48 \\
\hline L Lingual Gyrus & & & 3.87 & -44 & -42 & -44 \\
\hline
\end{tabular}

Table 4. Areas whose activation survived FSL cluster correction $(z=3.2, p<0.001)$ for contrasts $a)$ (Second offer-First offer) and b) (First offer-Second offer). The coordinates are anatomical MNI, and the areas have been identified with FSL Harvard-Oxford atlas. 


\begin{tabular}{ccccc}
\hline \multicolumn{5}{c}{ Connectivity (anatomical VMPFC seed) } \\
\hline Peak & Voxels & $\mathbf{x}$ & $\mathbf{y}$ & $\mathbf{z}$ \\
\hline R Superior Parietal Lobule & 3508 & 19 & -69 & 55 \\
\hline L Insula & 399 & -37 & -16 & -1 \\
\hline L Inferior Parietal Lobule & 291 & -40 & -41 & 41 \\
\hline Cingulate Gyrus, Anterior Dorsal & 240 & 9 & 12 & 37 \\
\hline L Middle Frontal Gyrus (BA 46) & 169 & -33 & 40 & 13 \\
\hline L Middle Frontal Gyrus (BA 6) & 107 & -26 & -2 & 55 \\
\hline
\end{tabular}

Table 5. Areas whose activation survived 3dClust Sim estimation for the connectivity with $V M P F C$ at the moment of the presentation of the second offer $(F W E=0.05, p$ (uncorrected $)=$ 0.0007). The coordinates are MNI, and the anatomical regions have been identified with AFNI atlas. 
$2 \mathrm{sec}$

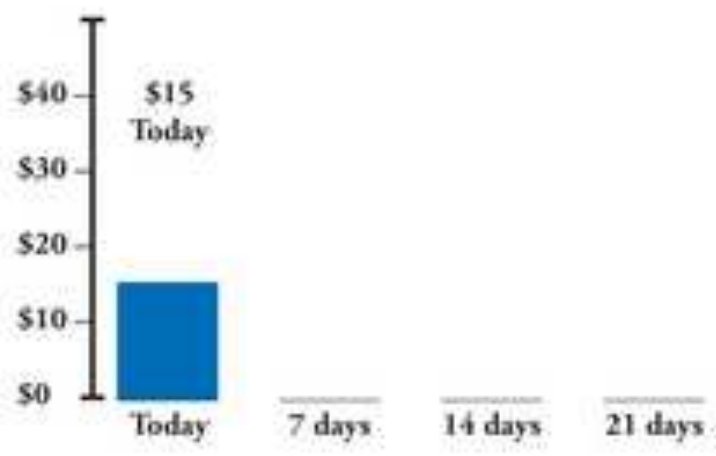

First offer
$2 \mathrm{sec}$

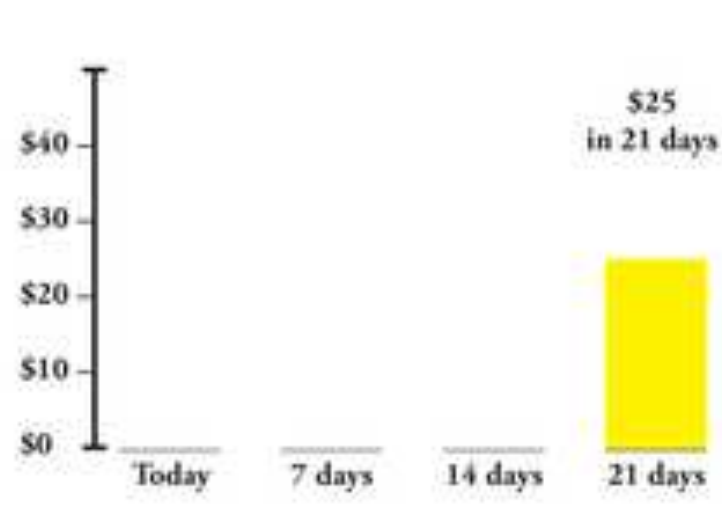

Second offer

\section{RT}

Please choose

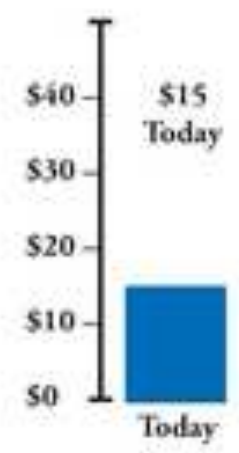

7 days

14 days 
A

Subjective value, offer 1

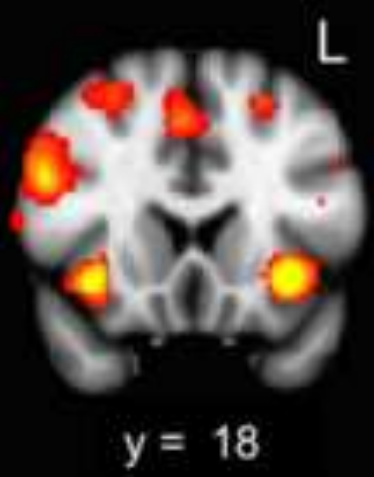

B

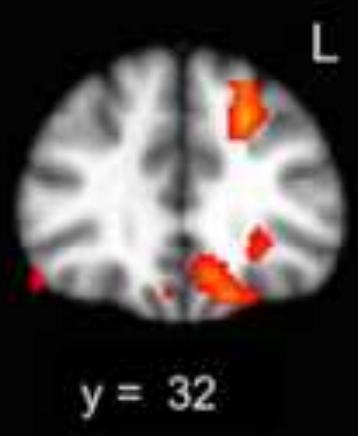

C

Contrast 02-01

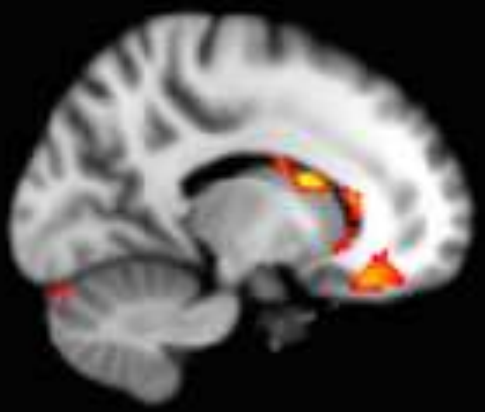

$$
x=-14
$$

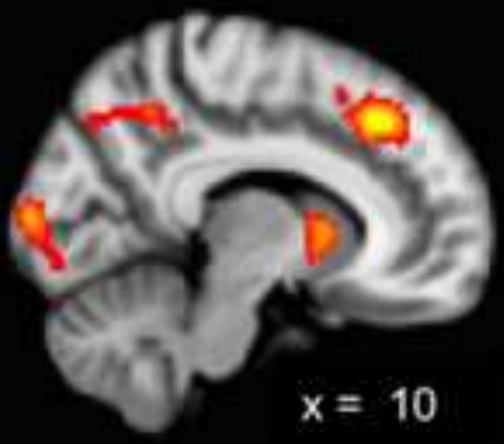

Subjective value, offer 2

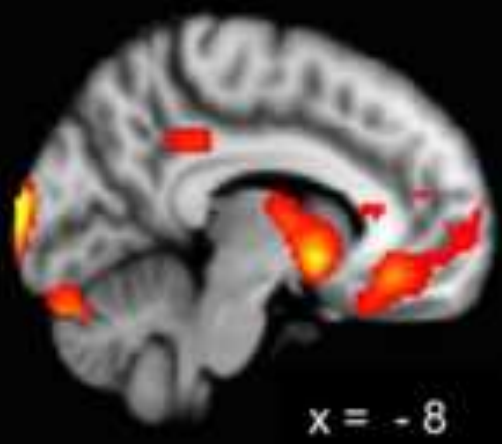

Contrast 01-02

D

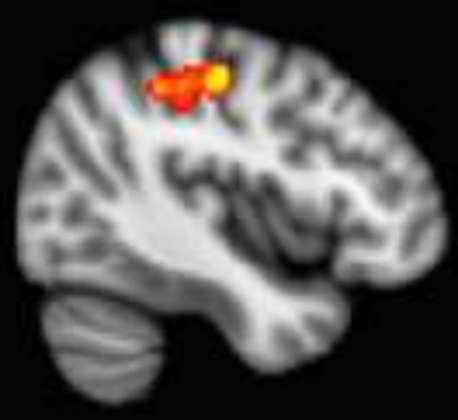

$$
x=-42
$$



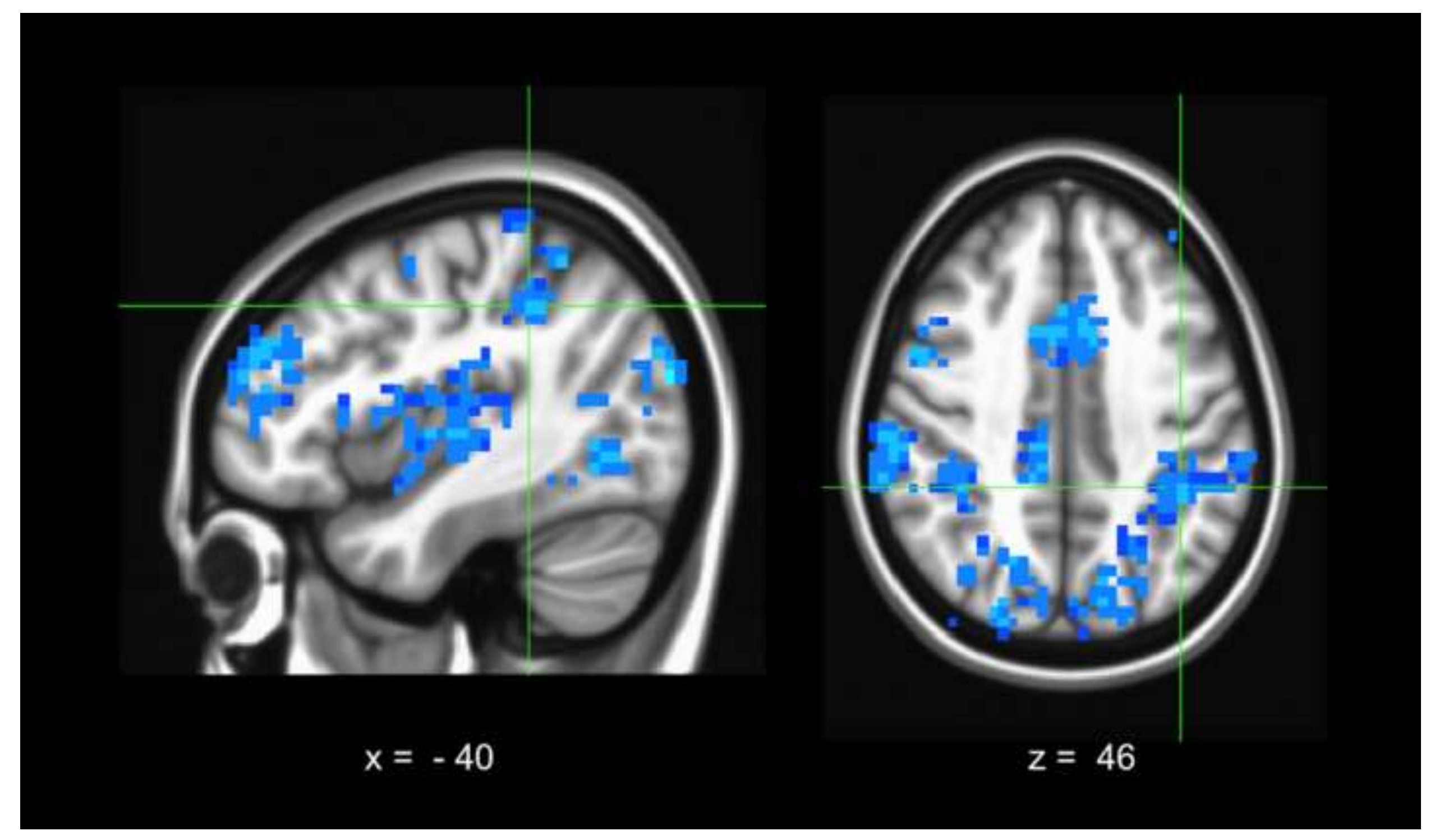

$$
x=-40
$$$$
z=46
$$ 